\title{
On hold: what to report after a study is halted?
}

\begin{abstract}
A $s$ any anesthetist or anesthesiologist knows, there is always a risk of complications when a patient is put under general anesthesia. This risk became reality when Dr. Giorgio Ionnellis dog died while undergoing an experimental cardiac surgical procedure. The veterinary technician administering and monitoring the isoflurane anesthesia tried, but she was unable to revive and save the animal when its blood pressure dropped acutely, and the animal went into cardiac arrest.

Ionnelli voluntarily halted his study until the school's veterinarians and the IACUC could investigate the incident. After a thorough review, the investigators reported that they found no problems with the surgeon's performance, the technician's efforts to revive the dog, or the readouts from the blood pressure and electrocardiographic monitors. They suspected that the anesthetic vaporizer, which had been serviced recently, was providing an excessive amount of isoflurane gas at each setting of the machine. This
\end{abstract}

suspicion was confirmed after an inspection by another technician from the company that had serviced the vaporizer. The machine was repaired and recalibrated, but the IACUC was faced with the question of what to report to the federal government, if anything. Some IACUC members and Ionnelli believed this was a single instance of a mechanical failure and not noncompliance with the PHS Policy ${ }^{1}$ or the Guide ${ }^{2}$. However, the chairman of the IACUC said that because Ionnelli voluntarily halted his study, and the IACUC did not disagree with that action, the stoppage was analogous to a suspension by the IACUC and it had to be reported as such to OLAW and the USDA. The veterinarians were unsure of what advice to give to the IACUC. Although Ionnelli's IACUC-approved protocol clearly stated that after induction, anesthesia would be maintained at three percent isoflurane, and that was what was recorded on the anesthesia monitoring sheet, they knew that the numbers on the vaporizer showing the percent of isoflurane being delivered were not meant to be taken as the standard for judging the depth of anesthesia. Rather, they believed it was the job of the person monitoring the animal to adjust the anesthetic depth as needed.

What should the veterinarians tell the IACUC? What, if anything, should the IACUC report to OLAW and the USDA? Is there anything that might be done to help prevent a repeat of this problem?

\section{Jerald Silverman \\ University of Massachusetts Medical School, \\ Worcester, MA, USA. \\ e-mail: Jerald.Silverman@umassmed.edu}

Published online: 22 July 2019

https://doi.org/10.1038/s41684-019-0349-z

References

1. Office of Laboratory Animal Welfare. National Institutes of Health. Public Health Service Policy on Humane Care and Use of Laboratory Animals (US Department of Health and Human Services, Bethesda, Maryland, USA, 2015)

2. National Research Council. Guide for the Care and Use of Laboratory Animals. 8th edn. (The National Academies Press, Washington, DC, 2011)

\section{The benefits of reporting}

T his scenario describes equipment failure that unfortunately lead to the death of an animal. This falls under OLAW's Guidance on Prompt Reporting to OLAW under the PHS Policy on Humane Care and Use of Laboratory Animals ${ }^{1}$, which requires reporting of "conditions that jeopardize the health or wellbeing of animals, including natural disasters, accidents, and mechanical failures, resulting in actual harm or death to animals".

However, this scenario does not state whether the study is PHS funded; the IACUC would therefore need to refer to their Animal Welfare Assurance to determine their criteria for reporting to OLAW. If the study was not funded by PHS and the institution's Assurance states that only PHS-funded studies need be reported, then no reporting is required. However, if the Assurance is vaguely written, the institution should report this incident to OLAW. Under OLAW's Guidance, "Reporting promptly to OLAW under IV.F.3 serves dual purposes. Foremost, it ensures that institutions deliberately address and correct situations that affect animal welfare, PHS supported research, and compliance with the Policy. In addition, it enables OLAW to monitor the institution's animal care and use program oversight under the Policy, evaluate allegations of noncompliance, and assess the effectiveness of PHS policies and procedures".

Even though the IACUC investigation determined that this incident was not the result of a noncompliance or an animal welfare concern, there are benefits to reporting. The institution can describe how they have conscientiously addressed and corrected the issues related to this accident, including interviewing all personnel involved, having the machine re-inspected by its manufacturer, and reviewing the protocol to ensure compliance with actions taken during the surgery. By describing the investigative process, this demonstrates to OLAW that the institution is following processes outlined in their Animal Welfare Assurance and is committed to maintaining high standards in their program. Additionally, OLAW representatives can provide further guidance, if warranted, on follow up activities to prevent future issues.

Many institutions may see reporting to OLAW as a negative to their animal care and use program. They might for example worry about increased attention from activist groups or feel burdened by extra administrative work due to internal processes involved with reporting. But reporting can provide positive interactions with external regulatory agencies that are intended to assist institutions with maintaining compliance with animal welfare regulations. The veterinarians should recommend that the incident be reported to OLAW, including all the steps taken to ensure that appropriate processes were in place and functioning at the time of the event. The reporting of this incident should be discussed with the IACUC and the Institutional Officer as well.

Finally, a voluntary halt of studies is not the same as a suspension. A vote to suspend the protocol would require a convened IACUC meeting with a quorum of members. Therefore, this voluntary halt would not be reportable to the USDA.

Rebecca J. Spangenberg*, J. Michael Padron and Denise Ancharski-Stutler Children's Hospital of Philadelphia, Philadelphia, PA, USA.

*e-mail:SPANGENBER@EMAIL.CHOP.EDU

Published online: 22 July 2019

https://doi.org/10.1038/s41684-019-0352-4

References

1. Office of Laboratory Animal Welfare. National Institutes of Health. Guidance on Prompt Reporting to OLAW under the PHS Policy on Humane Care and Use of Laboratory Animals, NOTOD-05-034, February, 24, 2005. 